Article

\title{
Multi-relaxation time lattice Boltzmann simulations of oscillatory instability in lid-driven flows of 2D semi-elliptical cavity
}

\author{
Zhe FENG ${ }^{1}$ and Hee-Chang LIM $^{2,+}$ (D) \\ 1 School of Mechanical Engineering, Pusan National University, 2, Busandaehak-ro 63beon-gil, Geumjeong-gu, \\ Busan, 46241, Rep. of KOREA; hclim@pusan.ac.kr \\ * Correspondence:hclim@pusan.ac.kr, Tel.: +82 515102 302; Fax: +82 515125236
}

\begin{abstract}
In this study, the multi-relaxation-time lattice Boltzmann method is applied to investigate the oscillatory instability of lid-driven flows in two-dimensional semi-elliptical cavities with different vertical to horizontal aspect ratios $\mathrm{K}$ in the range of 1.0-3.0. The program implemented in this study is parallelized using CUDA (compute unified device architecture), a parallel computing platform, and computations are carried out on NVIDIA Tesla K40c GPU. To carry out precise calculations, the CUDA algorithm is extensively investigated, and its parallel efficiency indicates that the maximum speedup is 47.6 times faster. Furthermore, the steady-oscillatory Reynolds numbers are predicted by implementing the CUDA-based programs. The amplitude coefficient is defined to quantify the time-dependent oscillation of the velocity magnitude at the monitoring point. The simulation results indicate that the transition Reynolds numbers correlate negatively with the aspect ratio of the semi-elliptical cavity, and are smaller than those of the rectangular cavity at the same aspect ratio. In addition, the detailed vortex structures of the semi-elliptical cavity within a single period are also investigated when the Reynolds number is larger than the steady-oscillatory value to determine the effects of periodic oscillation of the velocity magnitude.
\end{abstract}

Keywords: lattice Boltzmann method; mass-conserved wall treatment; non-equilibrium extrapolation boundary condition; mass leakage; parallel computation; CFD

\section{Introduction}

In recent years, the lattice Boltzmann method (LBM) [1,2] has been proposed as a promising and suitable alternative to the conventional computational fluid dynamics (CFD) method, particularly for incompressible flows [3], porous-media flows [4], magnetohydrodynamics [5], single-phase and multiphase fluid flows [6]. Unlike the traditional CFD that solves the Reynolds averaged Navier-Stokes equations (RANS) to simulate macroscopic flows [7], the LBM models the fluid as fictive particles using discrete distribution functions [1], and these particles undergo consecutive collision and streaming processes over a discrete lattice mesh. Owing to its particulate nature and local dynamics, the LBM has several advantages over traditional CFD methods, particularly in terms of the intrinsic parallelism of algorithm and data structure [8], and the treatment of complex boundaries [9]. Moreover, the lattice Boltzmann equation can yield second-order accuracy in time and space discretization [2], which is comparable to the accuracy of finite-volume numerical solutions of Navier-Stokes equations.

In the past half-century, lid-driven cavity flow has been used as a classical benchmark problem to validate numerical methods for incompressible fluid flow. It exhibits almost all phenomena that possibly exist in incompressible flows: eddies, secondary flows, instabilities, chaotic particle motions, transition, and turbulence [10]. Moreover, it also plays an important role in many industrial applications, such as in flexible blade coaters and melt spinning processes used in the manufacture of microcrystalline material [11]. Early studies have focused mostly on two-dimensional square cavity flow owing to its relatively simple geometry and easy implementation of numerical models. Ghia 
et al.[7] and Schreiber \& Keller [12] were among the first to report steady flow calculations of this benchmark quality, which can be used to validate subsequent numerical methods. Studies were later conducted using different numerical methods. Kalita \& Gupta [13] used the finite difference method to formulate the governing equations in stream function-velocity variables. Wright \& Gaskell [14] applied the finite volume method for primitive variable formulation. They presented a second and fourth order spatially accurate steady solution for a range of Reynolds numbers between 100 and 1,000. Olson \& Tuann [15] modified the full Navier-Stokes equations in the form of a single fourth-order equation for stream function using the finite element method, such that the velocities were continuous, and the incompressibility was accurately satisfied. Owing to progress in computing techniques and numerical models, the LBM has emerged as a promising numerical method for complicated flow problems. Hou et al. [16] used the LBM with compressibility effects to simulate lid-driven cavity flow when the Reynolds number is smaller than 7,500. Similarly, Lin et al.[17] applied the multi-relaxation-time (MRT) LBM to compute a steady solution at various Reynolds numbers $(100-7,500)$. Their steady-state results show good agreement with those of Ghia et al.[7], which validates the results obtained using LBM for lid-driven cavity flow problems.

Most of the studies above are based on square cavity; they analysed the vortex structures within the cavity, and presented the velocity profiles at midplanes, which can be used for benchmark tests. Some researchers also studied flow structures in a rectangular cavity [17-19], which has a wider field of application. To extend existing knowledge on the flow inside a simple cavity, it is necessary to conduct a more in-depth study on cavity flow within more complicated geometries. Erturk \& Dursun [20] simulated skew cavity flow at different skew angles for Reynolds numbers of 100 and 1000. Duan \& Liu [21] studied triangular cavity flow using single-relaxation-time (SRT) LBM, and their results showed good agreement with results obtained from existing studies. Zhang et al. [22] investigated two-dimensional isosceles trapezoidal cavity flow using the SRT-LBM for Reynolds numbers and top angles in the range 100-15,000 and 50-90, respectively. In the simulation, it was found that as the Reynolds number increases, the flow undergoes a complicated transition from steady to periodic state, and finally to chaotic flow. In addition, Glowinski et al.[23] studied lid-driven incompressible viscous flow in two-dimensional semi-circular cavity using an operator-splitting/finite elements-based methodology. They obtained the velocity profiles at midplanes and measured the angles of detachment of the secondary and tertiary vortices, which can be used for reference in the benchmark tests. Moreover, it has been demonstrated that Hopf bifurcation occurs at approximately $\operatorname{Re}=6,600$ for semi-circular cavity flow. Mercan \& Atalik [24] studied lid-driven arc-shaped cavity flows at high Reynolds numbers by adopting the unsteady stream function-vorticity transport formulation. They investigated the effects of arc angle ratio on the formation and growth of vortex structures, and also discussed the existence and development of periodic solutions. Furthermore, Idris et al. [25] simulated lid-driven flow in semi-ellipse shallow cavities with aspect ratios of 1:4, 1:3 and 3:8 using the stream function-vorticity approach for Reynolds numbers in the range 100-2,000. Recently, Ren \& Guo [26] employed the SRT-LBM to simulate steady flow in two-dimensional lid-driven semi-elliptical cavity with Reynolds numbers and vertical to horizontal ratios (K) in the range 500-5,000 and 0.1-4, respectively. They investigated the effects of Re and K on the pressure field and vortex structures. From the above discussions, it can be observed that interest in lid-driven flows with complex geometries has increased significantly, which may lead to wider industrial applications in the future, comparable to rectangular cavity. In addition to the description and analysis of vortex structures in cavities, it is also necessary to find the critical Reynolds number of Hopf bifurcation, which is important to maintain the steady state of fluid flows. Some researchers have investigated the transition process of fluid flow in cavities with complex geometries, and the reported critical Reynolds numbers are obviously different from that of the normal rectangular cavity.

LBM is very suitable for making code parallelization because it has an explicit collision-streaming scheme and it updates its node with only the nearest neighbour nodes. This can be realized using a graphical processing unit (GPU) through the compute unified device architecture (CUDA) [? ]. Several 
researchers [28-30] have demonstrated the successful implementation of the LBM on GPUs and have reported that the computational efficiency of GPUs exceeds that of PC-based CPUs (i.e., cluster-based CPUs have higher efficiency).

In this study, a novel efficient CUDA MRT-LBM was implemented to investigate oscillatory instability in lid-driven flows of 2D semi-elliptical cavity. The parallel performance of the CUDA-based program was analysed, as well as the variation of vortex structures within the cavity with time. This paper is organized as follows: Chapter 2 outlines the computational methodology, including the governing equations, boundary conditions and parallel algorithm. Chapter 3 compares and analyses the numerical results. Finally, Chapter 4 presents the major conclusions.

\section{Design of LBM Simulation}

\subsection{Multi-relaxation-time LBM (MRT-LBM)}

In the MRT-LBM, the collision operator is represented in a matrix form. Compared with the SRT-LBM model, the MRT-LBM has better numerical stability, wider range of physical applications, and can be extended to non-isotropic flows [31]. The lattice Boltzmann equation (LBE) is given as

$$
f(\mathbf{x}+\mathbf{c} \triangle t, t+\triangle t)-f(\mathbf{x}, t)=-\mathbf{M}^{-1} \mathbf{S}\left(m(\mathbf{x}, t)-m^{e q}(\mathbf{x}, t)\right)
$$

where $M$ is a matrix that transforms the distribution function $f$ from the velocity space to the momentum space $m=\mathbf{M} f$ at $(\mathbf{x}, t), \mathbf{c}$ is the particle velocity, and $\mathbf{S}$ is the relaxation diagonal matrix. The transformation matrix is usually an orthogonal matrix, its inverse matrix can be calculated as follows

$$
\mathbf{L}=\mathbf{M} \mathbf{M}^{\mathrm{T}}, \mathbf{M}^{-1}=\mathbf{M}^{\mathrm{T}} \mathbf{L}^{-1}
$$

where $\mathbf{L}^{-1}$ can be onsidered as the matrix of rescaled factors. Based on the particle distribution functions, the macroscopic density and velocity are defined as

$$
\begin{gathered}
\rho=\sum_{i=0}^{N} f_{i} \\
\rho \mathbf{u}=\sum_{i=0}^{N} f_{i} \mathbf{c}_{\mathbf{i}}
\end{gathered}
$$

In this study, the D2Q9 lattice model was adopted as shown in Fig. 1. The lattice speeds can be written as

$$
\mathbf{c}_{\mathbf{i}}= \begin{cases}(0,0), & i=0, \\ c\left(\cos \left(\frac{(i-1) \pi}{4}\right), \sin \left(\frac{(i-1) \pi}{4}\right),\right. & i=1,2,3,4, \\ \sqrt{2} c\left(\cos \left(\frac{(i-1) \pi}{4}\right), \sin \left(\frac{(i-1) \pi}{4}\right),\right. & i=5,6,7,8\end{cases}
$$

The transformation matrix and the corresponding diagonal matrix of rescaled factors can be given as 


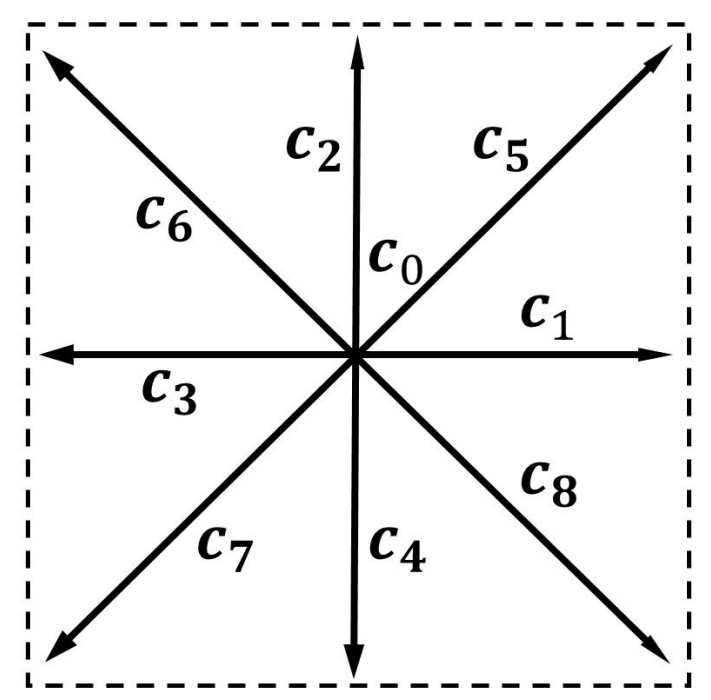

Figure 1. Schematic of the D2Q9 lattice model

$$
\mathbf{M}=\left(\begin{array}{ccccccccc}
1 & 1 & 1 & 1 & 1 & 1 & 1 & 1 & 1 \\
-4 & -1 & 2 & -1 & 2 & -1 & 2 & -1 & 2 \\
4 & -2 & 1 & -2 & 1 & -2 & 1 & -2 & 1 \\
0 & 1 & 1 & 0 & -1 & -1 & -1 & 0 & 1 \\
0 & -2 & 1 & 0 & -1 & 2 & -1 & 0 & 1 \\
0 & 0 & 1 & 1 & 1 & 0 & -1 & -1 & -1 \\
0 & 0 & 1 & -2 & 1 & 0 & -1 & 2 & -1 \\
0 & 1 & 0 & -1 & 0 & 1 & 0 & -1 & 0 \\
0 & 0 & 1 & 0 & -1 & 0 & 1 & 0 & -1
\end{array}\right)
$$

In the MRT-LBM, the moments [32] can be expressed as

$$
m=\left(\rho, e, \varepsilon, j_{x}, q_{x}, j_{y}, q_{y}, p_{x x}, p_{x y}\right)^{T}
$$

where $m_{0}=\rho$ is the density, $m_{1}=e$ is the kinetic energy, $m_{2}=\varepsilon$ is the square of the kinetic energy, $m_{3}=j_{x}$ and $m_{5}=j_{y}$ are the two components of the momentum, $m_{4}=q_{x}$ and $m_{6}=q_{y}$ are the two components of the energy flux, $m_{7}=p_{x x}$ and $m_{8}=p_{x y}$ are the stress tensors. The nine components of equilibrium in the moment space are

$$
m^{e q}=\left(\rho^{e q}, e^{e q}, \varepsilon^{e q}, j_{x}^{e q}, q_{x}^{e q}, j_{y}^{e q}, q_{y}^{e q}, p_{x x}^{e q}, p_{x y}^{e q}\right)^{T}
$$

where $m_{0}^{e q}=\rho$ is the mass density, the two components of the momentum $m_{3}^{e q}=j_{x}$ and $m_{5}^{e q}=j_{y}$ denote the conserved moments. Other non-conserved moments are given as

$$
\begin{cases}e^{e q}=-2 \rho+3\left(j_{x}^{2}+j_{y}^{2}\right), & \varepsilon^{e q}=\rho-3\left(j_{x}^{2}+j_{y}^{2}\right), \\ q_{x}^{e q}=j_{x}, & q_{y}^{e q}=-j_{y} \\ p_{x x}^{e q}=\left(j_{x}^{2}-j_{y}^{2}\right), & p_{x y}^{e q}=j_{x} j_{y}\end{cases}
$$

The diagonal relaxation matrix $\mathbf{S}$ is given by 


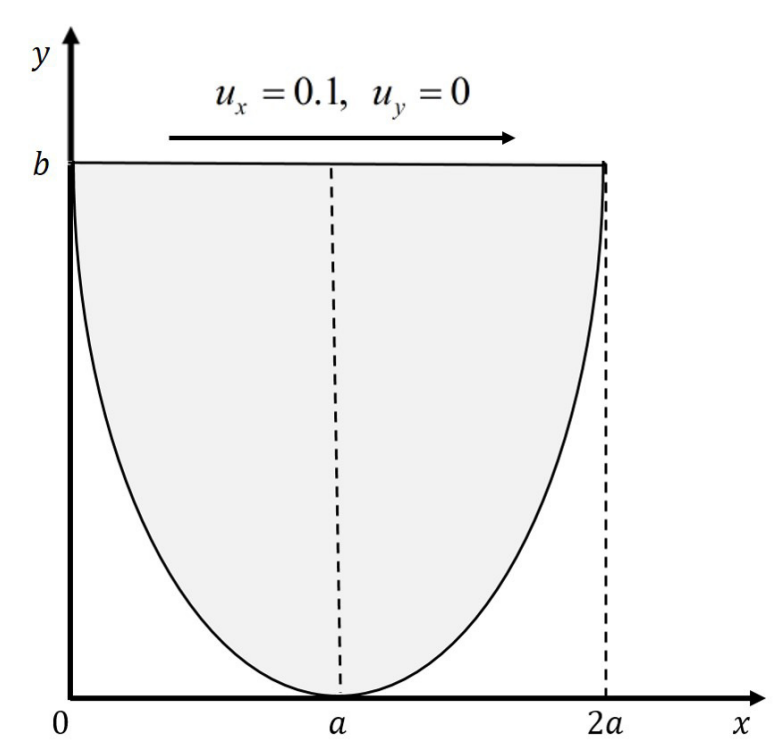

Figure 2. Schematic of the $2 \mathrm{D}$ semi-elliptical cavity

$$
\mathbf{S}=\operatorname{diag}\left(s_{0}, s_{1}, s_{2}, s_{3}, s_{4}, s_{5}, s_{6}, s_{7}, s_{8}\right)
$$

where $s_{0}=s_{3}=s_{5}=0$ to enforce mass conservation, and $s_{7}=s_{8}=1 / \tau$. Other elements are free parameters. To recover the corresponding SRT-LBM, all elements in the relaxation matrix should be set to $1 / \tau$. The relaxation time $\tau$ can be calculated from the kinematic viscosity $v$ as given below

$$
\tau=\frac{6 v+1}{2}
$$

\subsection{Calculation domain and boundary conditions}

The schematic of the 2D semi-elliptical cavity is shown in Fig. 2. The vertical to horizontal aspect ratio is defined as $K=b /(2 a)$. The position $(x, y)$ of fluid nodes can be described by the following equation:

$$
\frac{(x-a)^{2}}{a^{2}}+\frac{(y-b)^{2}}{b^{2}}<1,0<x<2 a, 0<y<b
$$

In this semi-elliptical cavity, the top wall is movable from left to right with a known constant velocity, while the semi-elliptical wall is stationary. The kinematic viscosity can be obtained from $R e=2 a U_{0} / v, U_{0}=0.1$. The free parameters in the relaxation matrix are set as $s_{1}=1.05, s_{2}=1.1, s_{4}=$ $s_{6}=1.25$ because the MRT-LBM model is used in this study. In addition, the convergence criterion is defined as

$$
\sum_{i, j}\left|\mathbf{u}_{i j}^{(n)}-\mathbf{u}_{i j}^{(n-100)} / \sum_{i, j}\right| \mathbf{u}_{i j}^{(n)} \mid<1.0 \times 10^{-8}
$$

Two types of boundary conditions exist for the above semi-elliptical cavity. Regarding the top wall, which moves at a constant velocity, the equilibrium distribution functions with a constant density are imposed along the top moving wall to accelerate LBM simulation to convergence as proposed by Hou et al. [16]. The equilibrium distribution function is defined as

$$
f_{i}^{e q}=\rho w_{i}\left[1+\frac{\mathbf{c}_{\mathbf{i}} \cdot \mathbf{u}}{c_{s}^{2}}+\frac{\left(\mathbf{c}_{\mathbf{i}} \cdot \mathbf{u}\right)^{2}}{2 c_{s}^{4}}-\frac{\mathbf{u} \cdot \mathbf{u}}{2 c_{s}^{2}}\right]
$$




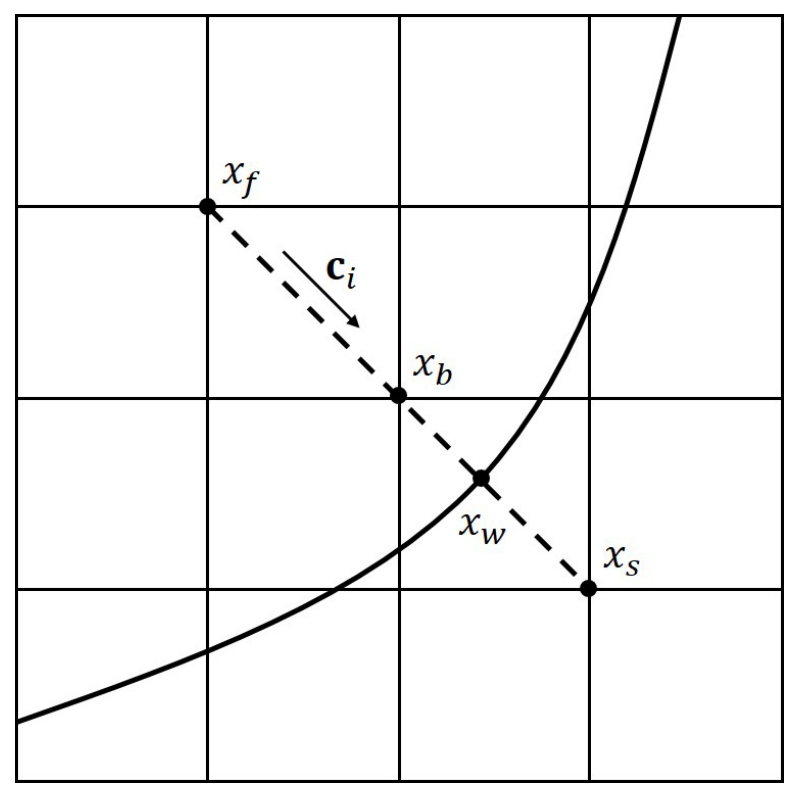

Figure 3. Schematic of the curved stationary wall

The distribution functions on the boundary nodes are not easily obtained because the semi-elliptical stationary wall is generally not aligned to the shape of the grid. Therefore, interpolation schemes should be used to predict these distribution functions. In this study, the interpolated bounce-back boundary condition proposed by Bouzidi et al. [32] is applied to handle the semi-elliptical stationary wall. As shown in Fig. 3 , the dashed line between the boundary node $x_{b}$ and the solid node $x_{s}$ intersects with the wall boundary at point $x_{w}$. Therefore, the lattice speed $\mathbf{c}_{\overline{\mathbf{i}}}$ denotes the reversed direction of $\mathbf{c}_{\mathbf{i}}$; thus, the fraction of the fluid domain in the dashed line can be defined as

$$
q=\left(\left|x_{b}-x_{w}\right|\right) /\left(\left|x_{b}-x_{s}\right|\right)
$$

In this study, linear interpolation is adopted to construct the unknown distribution functions at the boundary nodes, which are relevant to the known post-collision distribution functions at both the boundary nodes and the adjacent fluid nodes. The scheme is given as

$$
f_{\bar{i}}\left(x_{b}, t+\triangle t\right)= \begin{cases}2 q \tilde{f}_{i}\left(x_{b}, t\right)+(1-2 q) \tilde{f}_{i}\left(x_{f}, t\right), & q<\frac{1}{2} \\ \frac{1}{2 q} \tilde{f}_{i}\left(x_{b}, t\right)+\frac{2 q-1}{2 q} \tilde{f}_{\bar{i}}\left(x_{b}, t\right), & q \geq \frac{1}{2}\end{cases}
$$

\subsection{CUDA implementation}

In this study, CUDA-based MRT-LBM simulations were conducted using the NVIDIA Tesla K40c processor. Table 1 presents a summary of the specifications of the GPU hardware. The GPU system (the device) is a type of computational unit capable of executing multiple parallel execution threads; therefore, it functions as a co-processor to the main CPU (the host).

In November 2006, NVIDIA introduced CUDA, a general purpose parallel computing platform and programming model that leverages the parallel compute engine in NVIDIA GPUs to solve many complex computational problems more efficiently than a CPU [18]. It has extended the C language by allowing the programmer to define $C$ functions called kernels. They can be called from both the host and the device, but must be executed on the device. A kernel is defined using the _ global_ or the _ device__declaration specifier, and the number of CUDA threads that execute this kernel for a given kernel call is specified using a new $\langle\langle\langle\ldots\rangle\rangle\rangle$ execution configuration syntax. Each thread that executes the kernel is given a unique thread ID that is accessible within the kernel through the built-in threadId $x$ variable. 


\begin{tabular}{|c|c|}
\hline \multicolumn{2}{|c|}{ NVIDIA Tesla K40c } \\
\hline CUDA compute capability & 3.5 \\
Memory bus width (bits) & 384 \\
Momery clock rate (MHz) & 3004 \\
Global memory (MB) & 11440 \\
Constant memory (bytes) & 65536 \\
Shared memory per block (bytes) & 49152 \\
Maximum number of threads per block & 1024 \\
\hline
\end{tabular}

Table 1. Specifications of GPU hardware

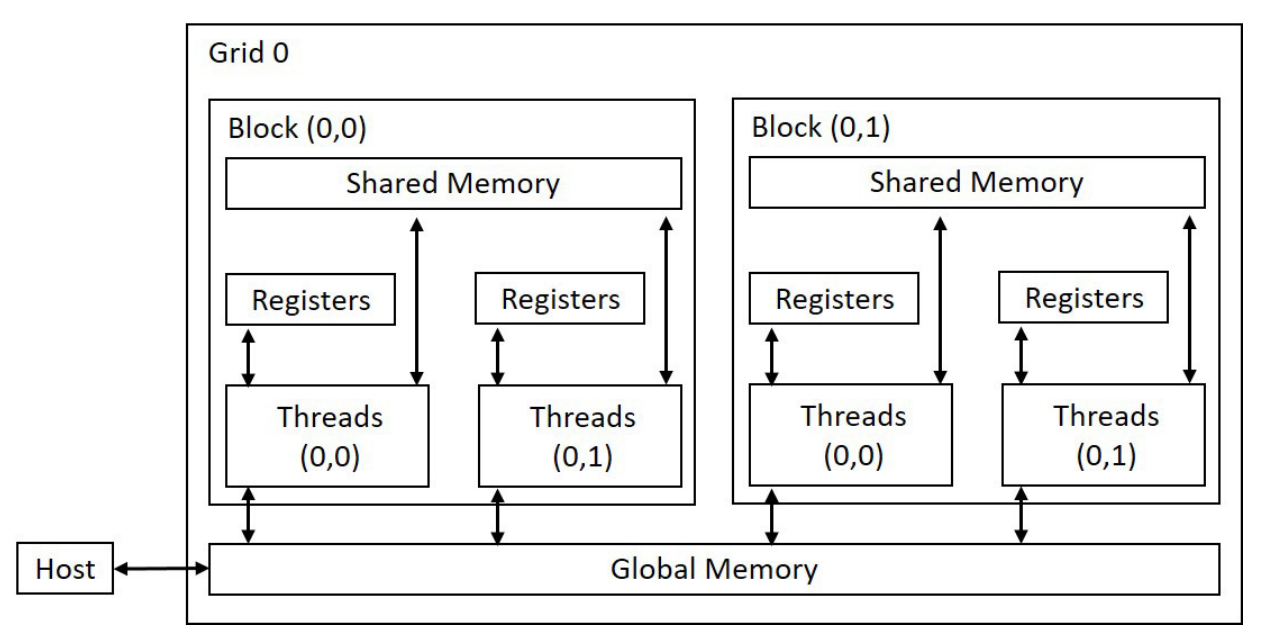

Figure 4. Memory spaces in the GPU

As the basic computing unit, CUDA threads may access data from multiple memory spaces during their execution as shown in Fig. 4. Each thread has a private local memory (i.e., register). In the same device, all threads have access to the global memory, which is the off-chip. It has the largest memory size as presented in Table 1, but has high latency for data access. Each thread block has shared memory visible to all threads of the block and with the same lifetime as the block. The shared memory has significantly higher bandwidth and lower latency than the local and global memory because shared memory is a type of on-chip memory. This memory freely allows data transfer between threads within the same block, but its memory size is small. In addition, the GPU hardware also has constant memory, which resides in the device memory and is cached in the constant cache.

As discussed above, shared memory has the lowest latency for data access, but its memory size is small. Global memory has the largest memory size, but with high data access latency. Therefore, optimization of data access is the most relevant issue regarding the parallel performance of LBM implementations in GPU using CUDA. The effects of using different memory spaces during computations will be presented in the next section. As shown in Fig. 5, the current LBM algorithm contains several processes, such as initialization, collision, streaming, boundary treatment for straight and curved walls, update of macroscopic variables, and output of the velocity and density.

In this CUDA algorithm, data transfer between the host and the device memory only occurs during the initialization and output processes. In the kernel functions executed in the GPU, computation data is frequently transferred between the global memory and shared memory. The detailed implementation of some functions is presented below.

In the initialization process, relevant data are declared in both the host memory and the device memory. The distribution functions, density, velocity and linking information (i.e., location of the boundary nodes and fraction of intersected points) of the curved boundary are initialized in the host memory, and copied from the host memory to the device memory. In the collision kernel, the post-collision distribution functions are calculated. From Eq. (1), it was found that rigorous matrix 


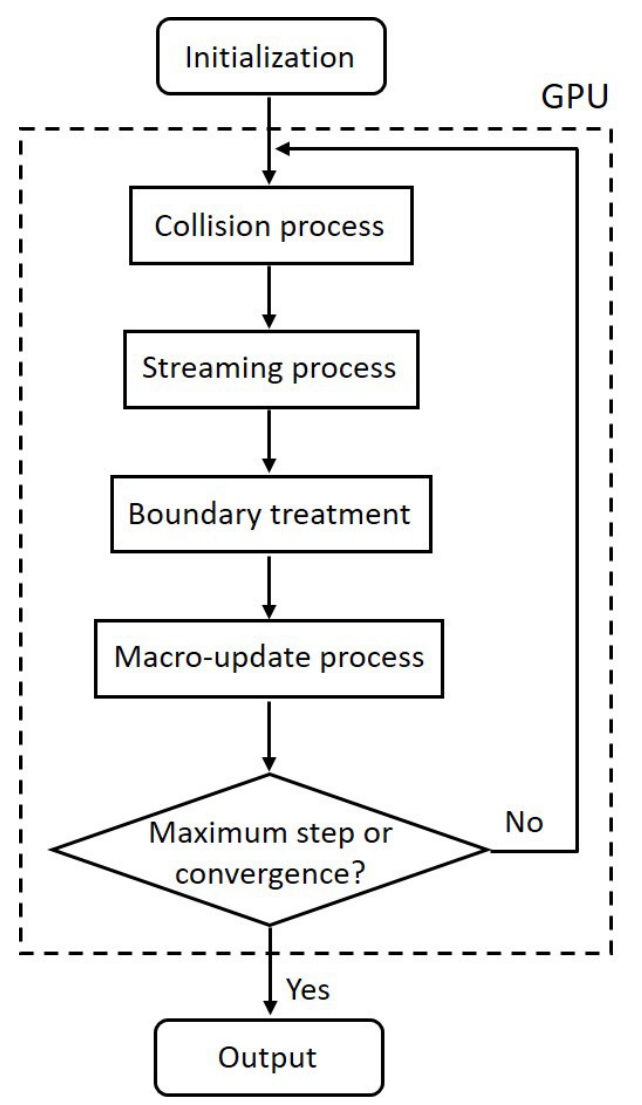

Figure 5. The CUDA algorithm for LBM

computations are needed to obtain these functions. To ensure efficient use of computational resources, all matrix manipulations are carried out in the shared memory. Therefore, the distribution functions (i.e., nine components), velocity (i.e., two components) and density should be declared in the shared memory; these functions use the double-precision floating-point data format, with a data size of 8 bytes. Although data access is very fast in shared memory, its memory size is small. The number of grid nodes (i.e., threads) allocated in each block should be carefully selected. In this study, the size of shared memory per block is 49,152 bytes as presented in Table 1 . There are 12 double-precision floating-point data variables (i.e., nine distribution function components, two velocity components and one density component) in each thread. Thus, the number of threads allocated in each block should not be greater than $49,152 /(12 \times 8)=512$.

In the streaming kernel function, each distribution function component will propagate to its adjacent node such that communication occurs between different blocks. It is unavoidable to access data through the global memory, which may affect parallel performance owing to its high latency. In the boundary treatment kernel, the focus is on the top straight wall and the side curved wall. It is implemented using the equilibrium boundary condition [16] and interpolated bounce-back boundary condition [32]. The kernel is implemented in the global memory because the number of boundary nodes is small.

In the kernel function of updating macroscopic variables (i.e., velocity and density), all computations are carried out in the shared memory, and they are used in the calculation of the next time step. In addition, the relative error of the velocity field is also computed in this kernel, which can be used to determine the convergence of the simulation. In the output process, LBM simulation has achieved its maximum time step or convergence state. The macroscopic variables are copied from the device memory to the host memory, so that they can be used for post processing. 


\begin{tabular}{c|c|c|c}
\hline \hline $\operatorname{Re}$ & Present & Glowinski et al. [23] & Ren \& Guo [26] \\
\hline \hline 500 & $(0.6350,0.3046)$ & - & $(0.6348,0.3047)$ \\
\hline 1,000 & $(0.6210,0.2953)$ & $(0.6214,0.2970)$ & $(0.6211,0.2949)$ \\
\hline 3,000 & $(0.6575,0.2973)$ & $(0.6514,0.2973)$ & $(0.6582,0.2969)$ \\
\hline 5,000 & $(0.6906,0.3062)$ & $(0.6833,0.3064)$ & $(0.6914,0.3066)$ \\
\hline \hline
\end{tabular}

Table 2. Comparison of center locations of primary vortex in the semi-circular cavity

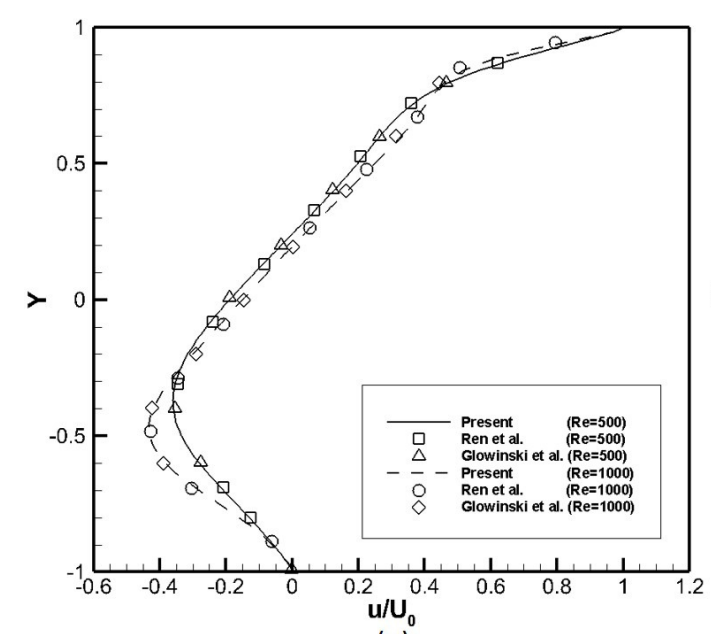

(a)

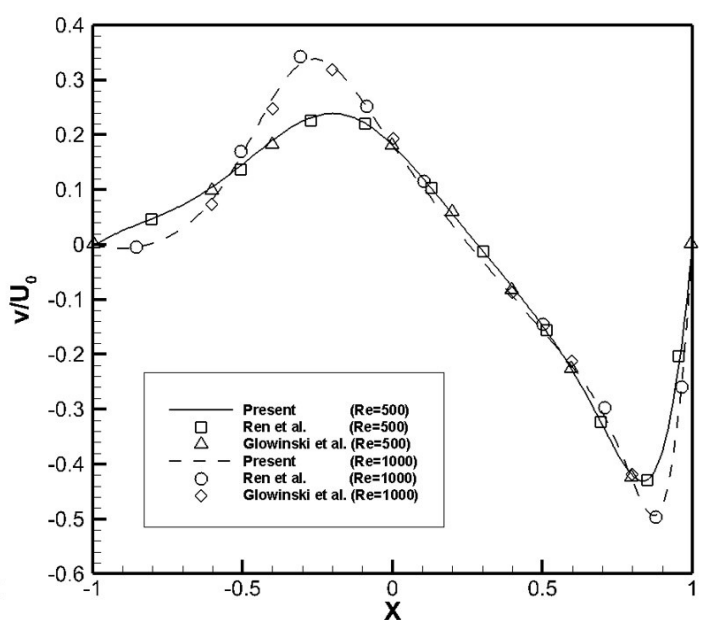

(b)

Figure 6. Horizontal and vertical velocity profiles along midplane of the semi-circular cavity: (a) $\operatorname{Re}=$ 500; (b) $\operatorname{Re}=1,000$

\section{Results and Discussion}

\subsection{Validation of the MRT-LBM}

For the purpose of validation, the MRT-LBM is used to investigate lid-driven flow in semi-circular cavity (i.e., semi-elliptical cavity with $\mathrm{K}=0.5$ ) at $\operatorname{Re}=500,1,000,3,000$ and 5,000, and the corresponding results are compared with those obtained in previous studies $[23,26]$. In the simulation, the number of lattice nodes in each axis was set to 257. Table 2 presents a comparison of the centre locations of the primary vortex in the semi-circular cavity. It was found that there is good agreement between the MRT-LBM results in this study and those obtained in previous studies. Furthermore, the horizontal velocity profiles along the midplane of the semi-circular cavity were also compared with those obtained in previous studies $[23,26]$ for $\operatorname{Re}=500$ and $\operatorname{Re}=1,000$. As depicted in Fig. 6, the two sets of results show good agreement. Therefore, the MRT-LBM proposed in this study can propertly simulate lid-driven flow in semi-elliptical cavities with different aspect ratios.

\subsection{Parallel performance}

This section presents the approach to develop and improve the performance of CUDA-based MRT-LBM program. Numerical simulations were carried out for lid-driven semi-elliptical cavity flow at Reynolds number $\mathrm{Re}=1,000$ and aspect ratio $\mathrm{K}=1.0$. Each numerical simulation was carried out using 50,000 iteration time steps and the wall time was logged to explore parallel performance. The CPU platform is on Intel Xeon E5, which was used as reference. GPU computations were carried out on NVIDIA Tesla K40c.

Two schemes are proposed to validate parallel performance because the GPU device has both global and shared memory. In parallel scheme 1, all computations were carried out in the global 


\begin{tabular}{c|c|c|c|c}
\hline \hline Wall time [s] & $128 \times 128$ & $256 \times 256$ & $512 \times 512$ & $1,024 \times 1,024$ \\
\hline \hline Intel Xeon E5 & 184.52 & 806.16 & $6,041.34$ & $24,322.31$ \\
\hline Parallel scheme 1 & 52.34 & 153.42 & 569.72 & $2,130.68$ \\
\hline Parallel scheme 2 & 19.96 & 42.78 & 149.73 & 511.43 \\
\hline \hline
\end{tabular}

Table 3. Elpased wall times for 50,000 iteration steps on CPU and GPU of various grid sizes

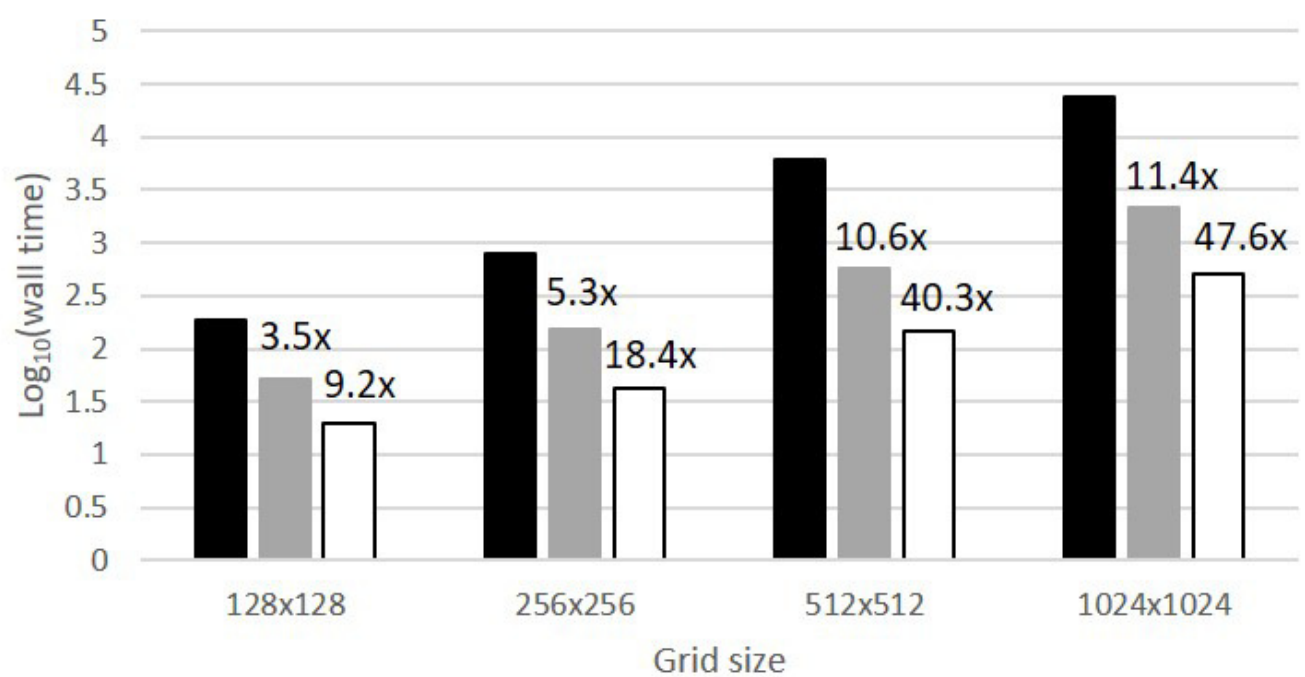

Intel Xeon E5 Parallel scheme $1 \quad$ aParallel scheme 2

Figure 7. Parallel performance of the CUDA program

memory, which means that only global memory was used in the kernel functions. In parallel scheme 2 , the parallel algorithm proposed in Section 2.3 was applied. Table 2 presents the elapsed wall time of the MRT-LBM simulations on CPU and GPU for various grid sizes. It was found that the elapsed time in parallel scheme 2 is smaller than that of scheme 1 for the same grid size. In addition, as shown in Fig.7, the parallel efficiency of GPU implementation is highly dependent on the grid size, which increases as the grid size increases. The speed-up of parallel scheme 2 is greater than that of parallel scheme 1, which indicates faster data access of the shared memory. In addition, the maximum GPU speed-up is 47.6 times faster than that of the CPU-based simulation in this study. Therefore, parallel scheme 2 was adopted for these simulations because rigorous numerical simulations need to be carried out to investigate oscillatory instability in lid-driven flows of semi-elliptical cavity.

\subsection{Oscillatory instability}

This section presents the numerical simulations carried out based on the MRT-LBM to identify oscillatory instability in lid-driven flows of 2D semi-elliptical cavity with aspect ratios of 1.0, 2.0 and 3.0. To determine the transition of fluid flow from steady state to oscillatory state, the magnitude of the velocity was continuously monitored from the start of the simulation. The location of this monitoring point is $(a / 2, b / 2)$. The time-dependent oscillation of the velocity magnitude is quantified by defining an amplitude coefficient.

$$
C_{a m p}=\frac{2\left(U_{\max }-U_{\min }\right)}{U_{\max }+U_{\min }}
$$




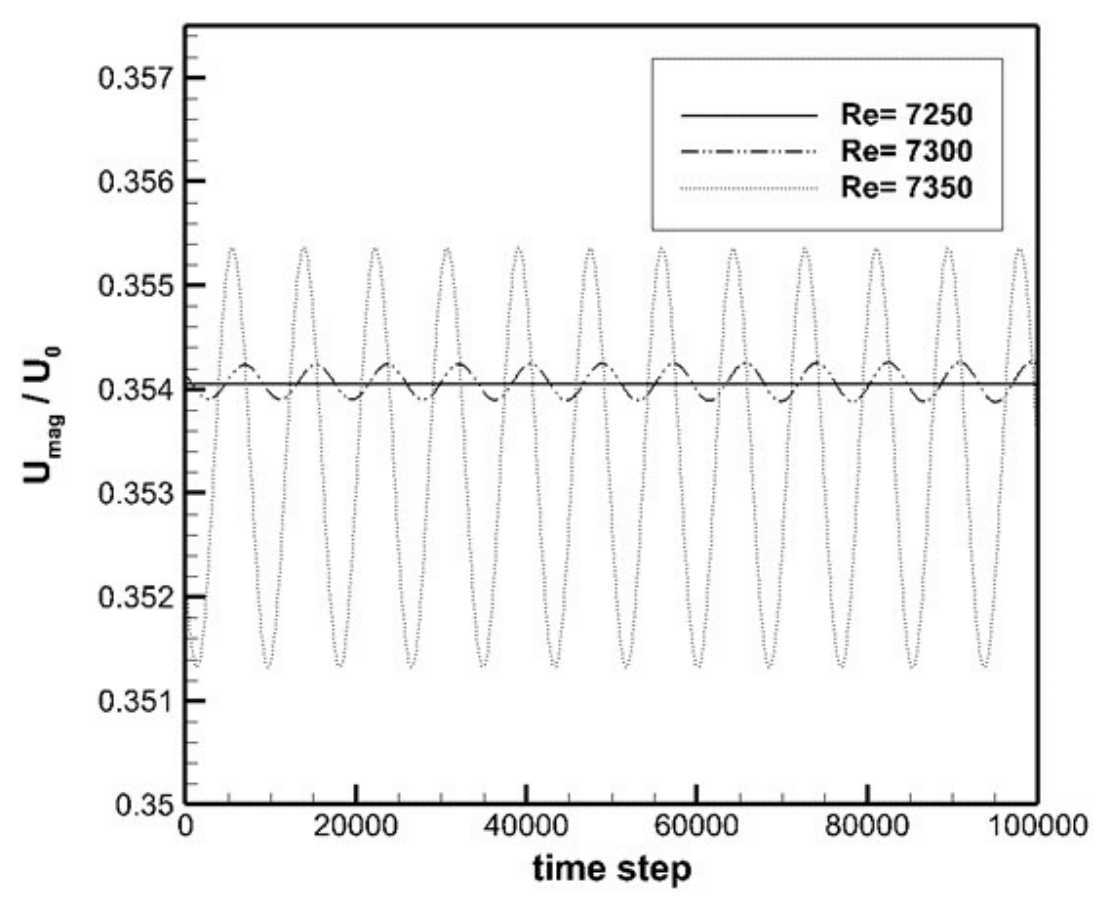

Figure 8. The variation of velocity magnitude at the monitor point for $\mathrm{K}=1.0$

where $U_{\max }$ and $U_{\min }$ are the maximum and minimum values of the velocity magnitude within one period of the time step. In this study, when the value of this amplitude coefficient is larger than $10^{-6}$, the flow state in the semi-elliptical cavity can be considered as oscillatory [28].

The time history (i.e., the last 100,000 steps of the entire simulation time) of the velocity magnitude measured at the monitoring point is shown in Figs. 8-10 for various Reynolds numbers. The velocity magnitude was normalized by the lid-driven surface velocity. As shown in Fig. 8, the variation of the velocity magnitude is almost steady at $\operatorname{Re}=7,250$. However, a small-amplitude oscillation occurs for $\operatorname{Re}=7,300$. Furthermore, as the Reynolds number increases to 7,350, the amplitude of oscillation increases. Similar trends are also observed for aspect ratios of 2.0 and 3.0.

Based on these time histories of the velocity magnitude, the amplitude coefficients are calculated for the above indicated Reynolds numbers as listed in Table 3. For $K=1.0$, it was found that the amplitude coefficient increases to 1.09E-3 when the Reynolds number increases to 7,300, whereas it decreases to $7.79 \mathrm{E}-7$ at $\operatorname{Re}=7,250$. This indicates that the steady-oscillatory Reynolds number for semi-elliptical cavity with $\mathrm{K}=1.0$ is in the range $7,250-7,300$. When the aspect ratio is 2.0 , the steady-oscillatory Reynolds number is in the range 5,650-5,700. At $\mathrm{K}=3.0$, the corresponding steady-oscillatory Reynolds number lies in the range 5,200-5,250. Moreover, it was found that the steady-oscillatory transition Reynolds number correlates negatively with the aspect ratio of the semi-elliptical cavity, which is consistent with solutions obtained in the two-dimensional rectangular cavity.

In addition to the prediction of the steady-oscillatory Reynolds numbers for semi-elliptical cavity at different aspect ratios, the solutions were also compared with those obtained for rectangular cavities at the same aspect ratios [28]. As a reference, the steady-oscillatory Reynolds numbers of rectangular cavity are also estimated by carrying out LBM simulations. Table 4 indicates that the steady-oscillatory Reynolds number of semi-elliptical cavity is smaller than that of rectangular cavity at the same aspect ratio, which is possibly due to the effect of cavity geometry.

From the above discussions, it can be observed that the oscillation of the velocity magnitude is periodic when the Reynolds number is larger than the steady-oscillatory value, which may have some effects on the vortex structure of the semi-elliptical cavity. The detailed vortex structures within a 


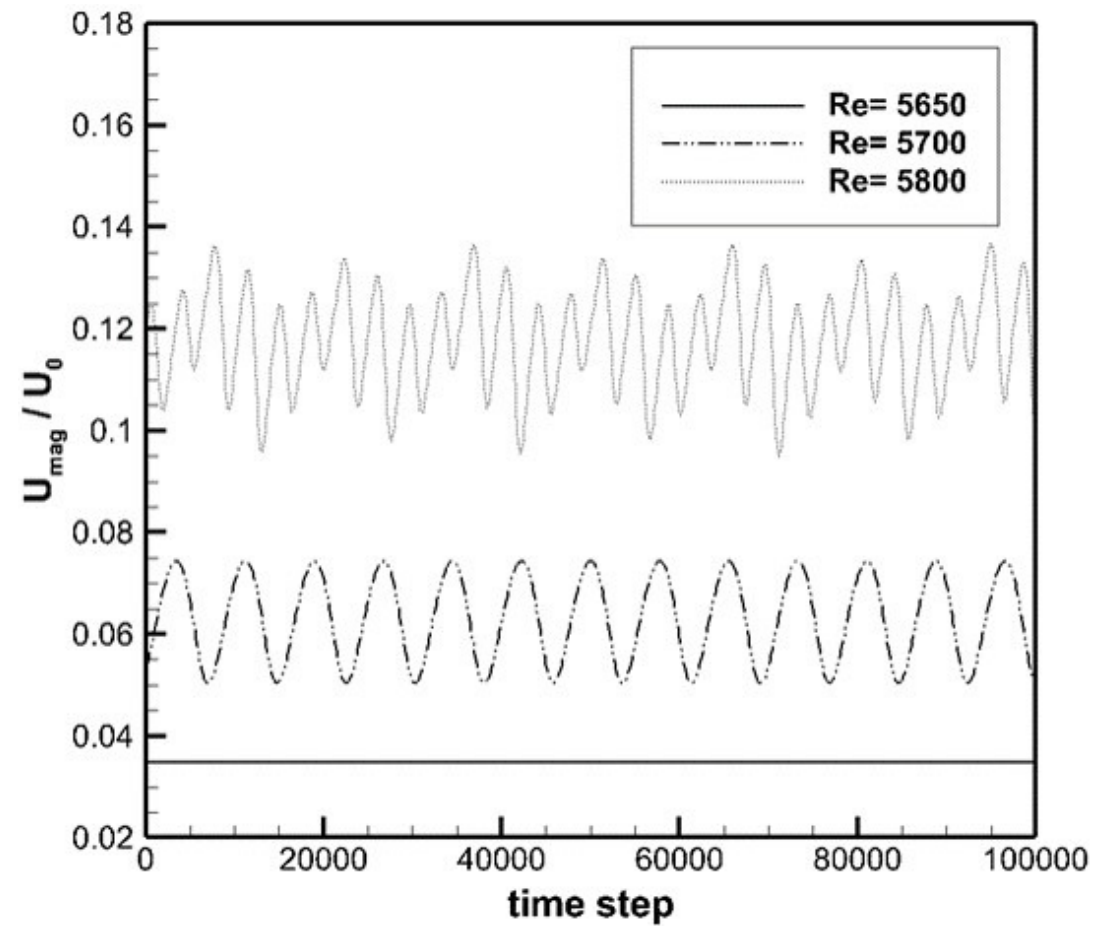

Figure 9. The variation of velocity magnitude at the monitor point for $\mathrm{K}=2.0$

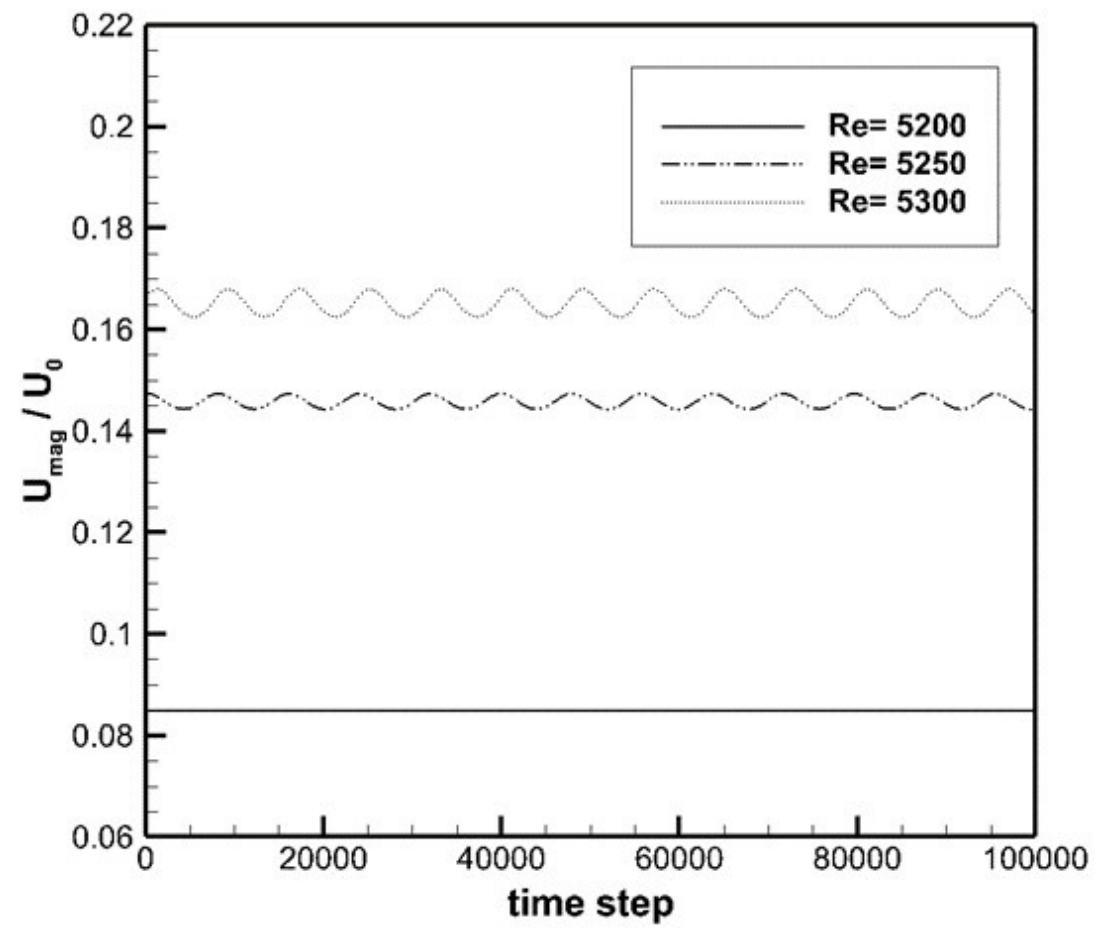

Figure 10. The variation of velocity magnitude at the monitor point for $K=3.0$ 


\begin{tabular}{|c|c|c|c|c|}
\hline \hline \multirow{2}{*}{$\mathrm{K}=1.0(512 \times 512)$} & $\operatorname{Re}$ & 7,250 & 7,300 & 7,350 \\
& $C_{a m p}$ & $7.79 \mathrm{E}-7$ & $1.09 \mathrm{E}-3$ & $1.14 \mathrm{E}-2$ \\
\hline \multirow{2}{*}{$\mathrm{K}=2.0(512 \times 1,024)$} & $\operatorname{Re}$ & 5,650 & 5,700 & 5,800 \\
& $C_{a m p}$ & $8.77 \mathrm{E}-7$ & $3.86 \mathrm{E}-1$ & $3.61 \mathrm{E}-1$ \\
\hline \multirow{2}{*}{$\mathrm{K}=3.0(512 \times 1,536)$} & $\operatorname{Re}$ & 5,200 & 5,250 & 5,300 \\
& $C_{a m p}$ & $1.53 \mathrm{E}-8$ & $2.12 \mathrm{E}-2$ & $3.33 \mathrm{E}-2$ \\
\hline \hline
\end{tabular}

Table 4. Amplitude coefficients for different Reynolds numbers and aspect ratios

\begin{tabular}{c|c|c|c}
\hline \hline Transition Re ranges & $\mathrm{K}=1.0$ & $\mathrm{~K}=2.0$ & $\mathrm{~K}=3.0$ \\
\hline \hline Semi-elliptical cavity & $7,250-7,300$ & $5,650-5,700$ & $5,200-5,250$ \\
\hline Rectangular cavity [28] & $8,325-8,350$ & $6,025-6,050$ & $5,725-5,750$ \\
\hline \hline
\end{tabular}

Table 5. Transition Re ranges for semi-elliptical cavity and rectangular cavity with different aspect ratios

single period T are presented in Figs. 11-13 for different aspect ratios, where $t_{0}$ is an arbitrary time step within this period. For $\mathrm{K}=1.0$, splitting and merging of the top left vortices can be clearly observed. The bottom primary vortex is stretched when a smaller vortex emerges below it, and is restored when this smaller vortex disappears. Moreover, these variations are periodic, which is consistent with the periodic oscillation of the velocity magnitude. For of $K=2.0$ and $K=3.0$, the number of primary vortices increased to three and four, respectively. The bottom primary vortex retains almost the same structure within the period. The primary variations reside in the top left vortices, which split and merge periodically. 


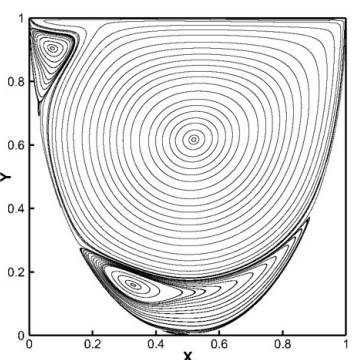

(1)

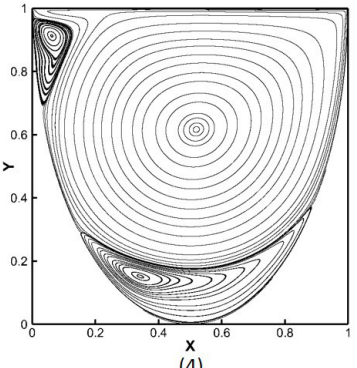

(4)

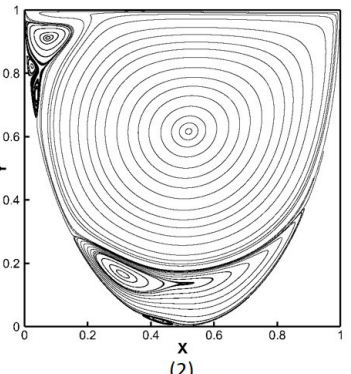

$x$
$(2)$

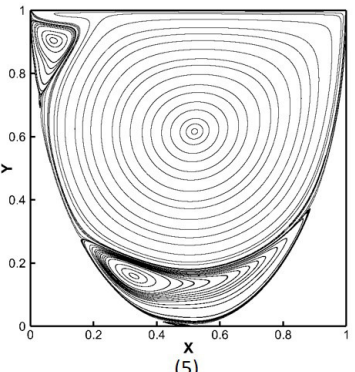

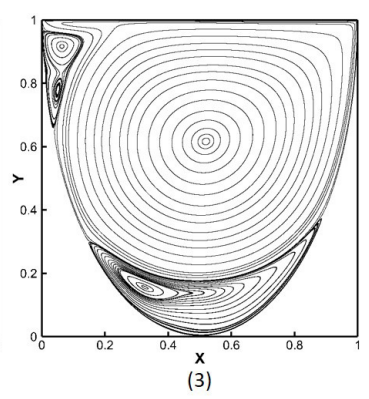

$(3)$

Figure 11. Variation of vortex structure within one period $\mathrm{T}$ at $\mathrm{K}=1.0$ and $\operatorname{Re}=7,350$ : (1) $t=t_{0}$; (2) $t=t_{0}+T / 4$; (3) $t=t_{0}+T / 2$; (4) $t=t_{0}+3 T / 4$; (5) $t=t_{0}+T$
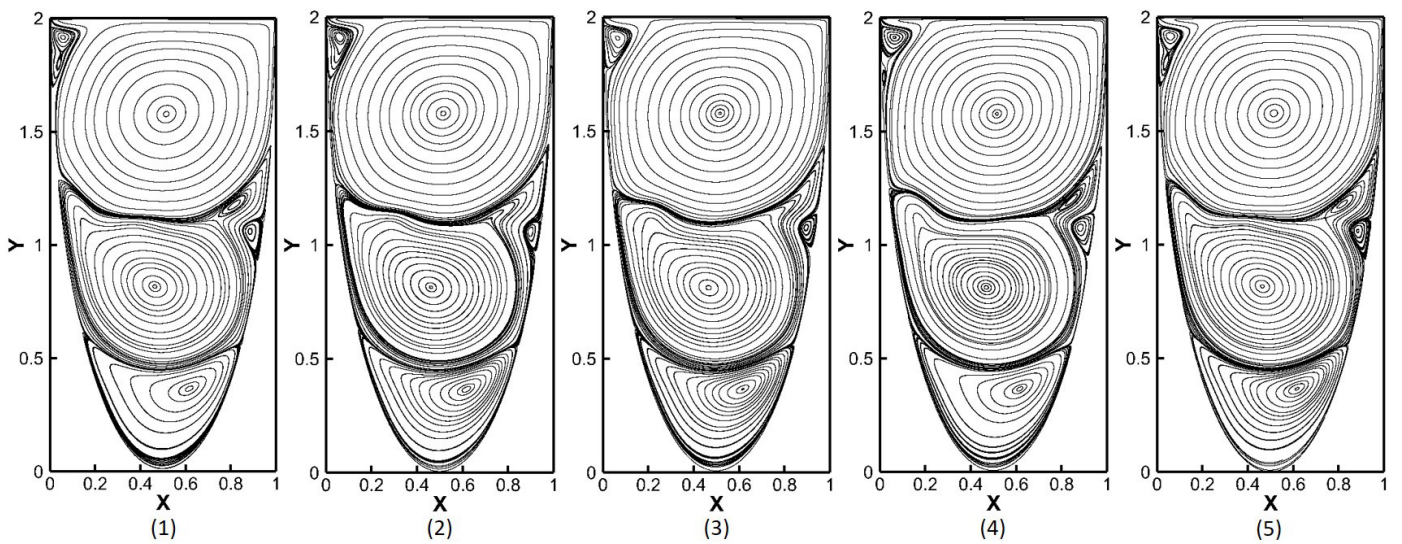

Figure 12. 12 Variation of vortex structure within one period $\mathrm{T}$ at $\mathrm{K}=2.0$ and $\operatorname{Re}=5700$ : $(1) t=t_{0} ;(2)$ $t=t_{0}+T / 4$; (3) $t=t_{0}+T / 2$; (4) $t=t_{0}+3 T / 4$; (5) $t=t_{0}+T$ 


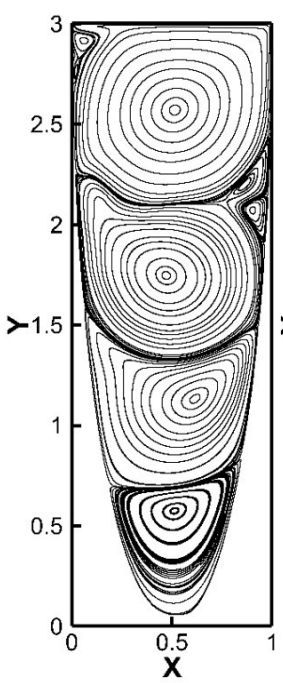

(1)

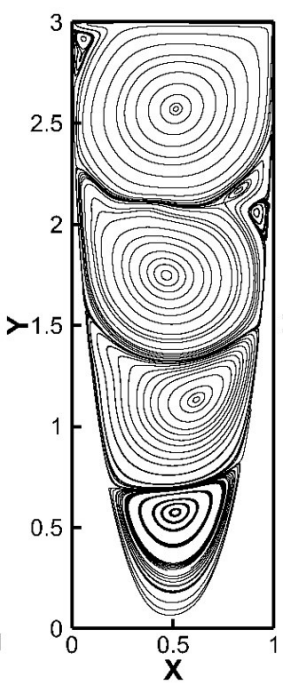

(2)

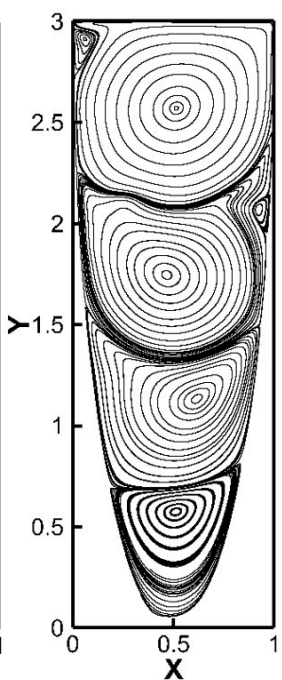

(3)

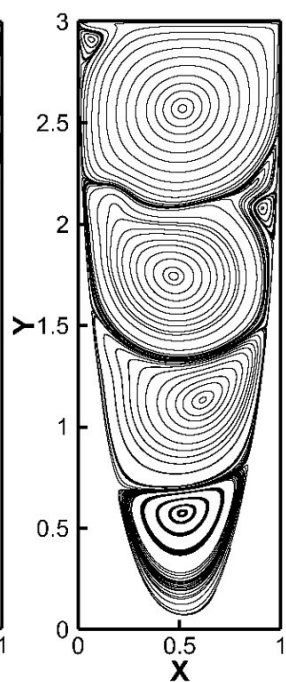

(4)

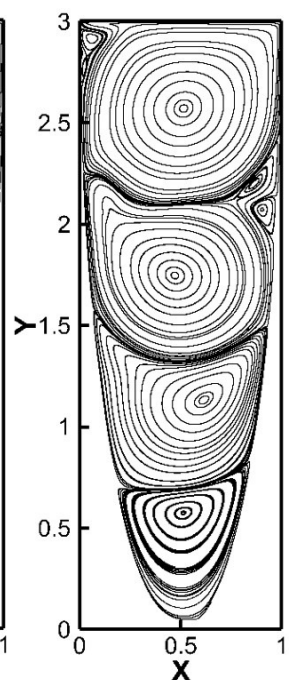

(5)

Figure 13. Variation of vortex structure within one period $\mathrm{T}$ at $\mathrm{K}=3.0$ and $\operatorname{Re}=5300$ : (1) $t=t_{0}$; (2) $t=t_{0}+T / 4$; (3) $t=t_{0}+T / 2$; (4) $t=t_{0}+3 T / 4 ;(5) t=t_{0}+T$

\section{Conclusion}

In this paper, the MRT-LBM was applied to investigate the steady-oscillatory transition of lid-driven flows in two-dimensional semi-elliptical cavity at three aspect ratios, namely, 1.0, 2.0 and 3.0. CUDA was used to parallelize the LBM program, and the computational platform was NVIDIA Tesla K40c GPU. In the current implementation of the CUDA program, shared memory was widely used in the kernel functions of the collision process and to update macroscopic variables, which resulted in a significantly higher parallel efficiency compared to using global memory. Moreover, the parallel efficiency of the CUDA program is highly dependent on the grid size. In this study, the maximum speedup of GPU implementation was 47.6 times faster than the same LBM simulation on Intel Xeon E5 CPU for a grid size of $1024 \times 1024$.

Several numerical simulations were carried out to predict the steady-oscillatory Reynolds number at different aspect ratios using the CUDA-based LBM program. An amplitude coefficient was defined to quantify the time-dependent oscillation of the velocity magnitude at the monitoring point $(a / 2, b / 2)$. For aspect ratios of $\mathrm{K}=1.0,2.0$ and 3.0, the steady-oscillatory Reynolds numbers were in the ranges of 7,250-7,300, 5,650-5,700 and 5,200-5,250, respectively. The Reynolds numbers correlate negatively with the aspect ratio of the semi-elliptical cavity. Moreover, these transition Reynolds numbers were smaller than those of the rectangular cavity at the same aspect ratio. In addition to the prediction of the steady-oscillatory Reynolds numbers, the detailed vortex structures of the semi-elliptical cavity were also presented at different aspect ratios. It was found that the primary variations were in the top left vortices, which split and merge periodically.

\section{Acknowledgements}

This work was supported by 'Human Resources Program in Energy Technology' of the Korea Institute of Energy Technology Evaluation and Planning (KETEP), granted financial resource from the Ministry of Trade, Industry \& Energy, Republic of Korea (no. 20164030201230). In addition, this work was supported by the National Research Foundation of Korea (NRF) grant funded by the Korea government (MSIP) (no. 2016R1A2B1013820). This research was also supported by the Fire Fighting Safety \& 119 Rescue Technology Research and Development Program funded by the Ministry of Public Safety and Security (MPSS-2015-79). 
1. Qian Y.H., d'Humieres D. \& Lallemand P., 1992, Lattice BGK model for Navier-Stokes equation. Europhys. Lett. 17, 479-484.

2. Chen S. \& Doolen G.D., 1998, Lattice Boltzmann method for fluid flow. Annu. Rev. Fluid Mech. 30, 329-364.

3. Wu J. \& Shu C., 2010, An improved immersed boundary-lattice Boltzmann method for simulating three-dimensional incompressible flows. J. Comput. Phys. 229, 5022-5042.

4. Hasert M., Bernsdorf J. \& Roller S., 2011, Lattice Boltzmann simulation of non-Darcy flow in porous media. Procedia Comput. Sci. 4,1048-1057.

5. Chen S., Chen H. \& Martinez D.O., Matthaeus W.H., 1991, Lattice Boltzmann model for simulation of magnetohydrodynamics. Phys. Rev. Lett. 67, 3776-3779.

6. Yan Y.Y., Zu Y.Q. \& Dong B., LAM, 2011, a useful tool for mesoscale modelling of single-phase and multiphase flow. Appl. Therm. Eng. 31, 649-655.

7. Ghia U., Ghia K.N. \& Shin C.T., 1982, High-Re solutions for incompressible flow using the Navier-Stokes equations and a multigrid method. Jour. Comput. Phys. 48, 387-411.

8. Shang Z., Cheng M. \& Lou J., 2014, Parallelization of Lattice Boltzmann method using MPI domain decomposition technology for a drop impact on a wetted solid wall. Int. Jour. Modelling, Simulation and Scientific Computing 5, 1350024.

9. Chang C., Liu C.H. \& Lin C.A., 2009, Boundary conditions for lattice Boltzmann simulations with complex geometry flows. Comp. Math. with Appl. 58, 940-949.

10. Shankar P.N. \& Deshpande M.D., 2000, Fluid Mechanics in the Driven Cavity. Annu. Rev. Fluid Mech. 32, 93-136.

11. Aidun C.K., Triantafillopoulos N.G. \& Benson J.D., 1991, Global stability of a lid-driven cavity with through flow: flow visualization studies. Phys. Fluids 3, 2081-2091.

12. Schreiber R. \& Keller H.B., 1983, Driven cavity flows by efficient numerical techniques. Jour. Comput. Phys. 49, 310-333.

13. Kalita J.C. \& Gupta M.M., 2010, A streamfunction-velocity approach for 2D transient incompressible viscous flows. Int. Jour. Numer. Meth. Fluids 62, 237-266.

14. Wright N. \& Gaskell P., 1995, An efficient multigrid approach to solving highly recirculating flows. Comput. Fluids 24, 63-79.

15. Olson M.D. \& Tuann S.Y., 1979, New finite element results for the square cavity. Comput. Fluids 7, 123-135.

16. Hou S., Zou Q., Chen S., Doolen G. \& Cogley A.C., 1995, Simulation of cavity flow by the lattice Boltzmann method. Jour. Comput. Phys. 118, 329-347.

17. Lin L.S., Chen Y.C. \& Lin C.A., 2011, Multi relaxation time lattice Boltzmann simulations of deep lid driven cavity flows at different aspect ratios. Comput. Fluids 45, 233-240.

18. Cheng M. \& Hung K.C., 2006, Vortex structure of steady flow in a rectangular cavity. Comput. Fluids 35, 1046-1062.

19. Patil D.V., Lakshmisha K.N. \& Rogg B., 2006, Lattice Boltzmann simulation of lid-driven flow in deep cavities. Comput. Fluids 35, 1116-1125.

20. Erturk E. \& Dursun B., 2007, Numerical solutions of 2-D steady incompressible flow in a driven skewed cavity. Jour. Appl. Math. Mech. 87, 377-392.

21. Duan Y.L. \& Liu, R.X., 2007, Lattice Boltzmann simulations of triangular cavity flow and free-surface problems. Jour. Hydrodyn. Ser. B 19, 127-134.

22. Zhang T., Shi B.C. \& Chai Z.H., 2010, Lattice Boltzmann simulation of lid-driven flow in trapezoidal cavities. Comput. Fluids 39, 1977-1989.

23. Glowinski R., Guidoboni G. \& Pan T.W., 2006, Wall-driven incompressible viscous flow in a two-dimensional semi-circular cavity. Jour. Comput. Phys. 216, 76-91.

24. Mercan H. \& Atalik K., 2009, Vortex formation in lid-driven arc-shape cavity flows at high Reynolds numbers. Eur. Jour. Mech. B/Fluid. 28, 61-71.

25. Idris M.S., Irwan M.A.M. \& Ammar N.M.M., 2012, Steady state vortex structure of lid driven flow inside shallow semi ellipse cavity. Jour. Mech. Eng. Sci. 2, 206-216.

26. Ren J.J. \& Guo P., 2017, Lattice Boltzmann Simulation of Steady Flow in a Semi-Elliptical Cavity. Commu. Comput. Phys. 21, 692-717. 
27. CUDA C Programming Guide 9.0, 2017, http://docs.nvidia.com/cuda/cuda-c-programming-guide/index.html.

28. Lin L.S., Chang H.W. \& Lin C.A., 2013, Multi relaxation time lattice Boltzmann simulations of transition in deep 2D lid driven cavity using GPU. Computers $\mathcal{E}$ Fluids 80, 381-387.

29. Hong P.Y., Huang L.M., Lin L.S. \& Lin C.A., 2015, Scalable multi-relaxation-time lattice Boltzmann simulations on multi-GPU cluster. Computers \& Fluids 110, 1-8.

30. Li C.G. \& Maa J.P.Y., 2017, Multi-relaxation-time lattice Boltzmann simulations of lid driven flows using graphics processing unit. Appl. Math. Mech.-Engl. Ed. 38, 707-722.

31. Kuzmin A. \& Mohamad A.A., 2008, Multi-Relaxation Time Lattice Boltzmann Model for Multiphase Flows. Inter. Jour. Mod. Phys. C. 19, 875-902.

32. Bouzidi M., Firdaouss M. \& Lallemand P., 2001, Momentum transfer of a Boltzmann-lattice fluid with boundaries. Phys. Fluids 13, 3452-3459. 